Article

\title{
Surface Parameters Retrieval from Fully Bistatic Radar Scattering Data
}

\author{
Ying Yang ${ }^{1,2}$, Kun-Shan Chen ${ }^{1,3, * \mathbb{C}}$ and Guofei Shang ${ }^{3}$ \\ 1 State Key Laboratory of Remote Sensing Science, Institute of Remote Sensing and Digital Earth, \\ Chinese Academy of Sciences, Beijing 100101, China; yangying@radi.ac.cn \\ 2 University of Chinese Academy of Sciences, Beijing 100190, China \\ 3 School of Land Resources and Urban and Rural Planning, Hebei GEO University, \\ Shijiazhuang 050031, China; shangguofei@hgu.edu.cn \\ * Correspondence: chenks@radi.ac.cn
}

Received: 1 February 2019; Accepted: 7 March 2019; Published: 12 March 2019

\begin{abstract}
Fully bistatic radar scattering from rough surfaces is of vital importance in terrain remote sensing, but results in bulky data volume. The scattering is dependent on physical parameters of the media and is controlled by the radar observation geometry. Together, the two sets of parameters determine the scattering patterns in a bistatic plane confined by incident and polar angles in both incident and scattering directions. For radar remote sensing, it is desirable to infer surface parameters of interest, with satisfactory accuracy, from large volumes of measured data sets. This is essentially a task of data mining. In this paper, we present model-generated bistatic radar scattering data, followed by a sensitivity analysis, to identify a suitable configuration in terms of parameter inversion from fully bistatic measurements by a Kalman filter-trained dynamic learning neural network (DLNN). Results indicate that with bistatic observation, superior retrieval performance (as compared to backscattering observation) can be readily achieved.
\end{abstract}

Keywords: bistatic radar scattering; random media; parameter inversion; neural network

\section{Introduction}

Radar scattering from a rough surface is a complex process and is widely applied in the remote sensing of terrain and sea [1-4]. Recently, interest has grown in bistatic observation. Global Navigation Satellite System-Reflectometry (GNSS-R) is one such application for remote sensing of the earth $[5,6]$. Bistatic configurations can provide desirable performance in various geophysical parameter observations and retrieval from microwave scattering measurements. Multi-angle observations can produce large amounts of data. Therefore, it is difficult to tackle the complex inversion problems from fully bistatic observations of rough surface. Extensive studies show that by analyzing the sensitivity of scattering in response to surface parameters, it has been a common practice to retrieve the geophysical parameters of interest, both geometric and dielectric, from the radar measurements that can be inverted. The commonly used approach is to use traditional statistical techniques (e.g., multiple linear regression analyses) [7-10]. Statistical approaches will perform well if the distribution function of the parameters to be inverted is known. However, in practice, such distribution functions are generally not known [11]. Furthermore, the traditional statistical techniques have difficulty dealing with some specific nonlinear problems. Another inversion method, a neural network, has been widely applied [12-18] because it has an intrinsic ability to generalize, and makes a weaker assumption about the statistics of the input data. The most valuable feature here is that the neural network is capable of forming highly nonlinear decision boundaries in the feature space [15]. 
If one perceives radar remote sensing-a stochastic electromagnetic wave scattering problem-more closely and deeply, the following the $4 \mathrm{Vs}$ characteristics may be recognized: volume, variety, velocity, and veracity. As pointed out by Hey et al. [19], the data science in the big data era combines and synergizes the observation, model prediction, and numerical simulation such that data transforms into information, and knowledge can be assured. Within radar imaging, as we see from the $4 \mathrm{Vs}$ above, more advanced data analytics apparently should be developed to explore richer information offered by fully bistatic radars with fully polarized remote sensing data, which is the main objective of this paper. Hence, it can be realized that a better solution of inverting rough surface parameters is that, by knowing the scattering patterns, one may be able to detect the presence of undesired random roughness of a reflective surface (e.g., an antenna reflector), and thus accordingly, and perhaps effectively, devise a means to correct or compensate phase errors. Therefore, studying electromagnetic wave bistatic scattering from random surfaces is both theoretically and practically motivated.

In this paper, we present parameter inversions, by means of neural network, from fully bistatic radar scattering data. Performance is evaluated by comparing extensive simulated data within the reachable scope. In next section, the AIEM model is adopted as a working model to generate the fully bistatic radar scattering. Before proceeding, the AIEM model is validated by full-wave numerical simulations and experimental measurements to give confidence to its application, followed by a sensitivity analysis of bistatic scattering to root-mean-squared (RMS) height, correlation length, and moisture content. Section 3 describes the procedure of surface parameter retrieval from bistatic scattering data, while the inversion results are presented and discussed in Section 4. Finally, the concluding remarks are given in Section 5.

\section{Fully Bistatic Radar Scattering Data Generated by the AIEM Model}

\subsection{Validation of a Bistatic Scattering Model}

Figure 1 sketches the geometry of bistatic radar scattering from a rough surface. The incident and scattered wave vectors, are defined as

$$
\begin{aligned}
k_{x} & =k \sin \theta_{i} \cos \phi_{i}, k_{y}=k \sin \theta_{i} \sin \phi_{i}, k_{z}=-k \cos \theta_{i} \\
k_{s x} & =k \sin \theta_{s} \cos \phi_{s}, k_{s y}=k \sin \theta_{s} \sin \phi_{s}, k_{s z}=k \cos \theta_{s}
\end{aligned}
$$

where $k$ is the incident wave number in free space; $\theta_{i}$ and $\phi_{i}$ are the incident angle and azimuth angle of the transmitter, respectively; and $\theta_{s}, \phi_{s}$ are the scattering angle and azimuth angle of the receiver respectively. Notice that the backscattering direction is at $\theta_{s}=\theta_{i}, \phi_{s}=\phi_{i}+180^{\circ}$.

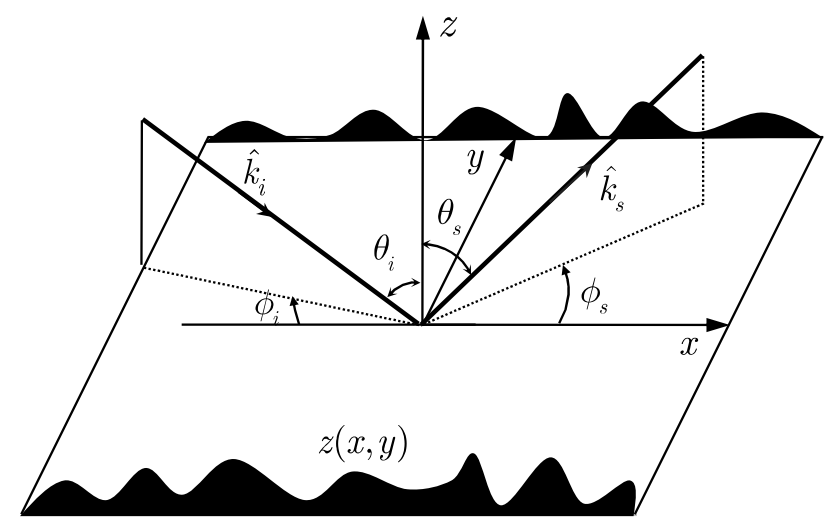

Figure 1. Geometry of bistatic radar scattering from a rough surface.

For the objective of this paper, we adopt the advanced integral equation method (AIEM) [20,21] that is based on a widely acceptable integral equation method (IEM) [2,3,22], an analytical model, to 
simulate the scattering coefficient in a fully bistatic mode. In an AIEM model, the scattering coefficient can be written as the sum of the single scattering $\sigma_{q p}^{0}(\mathrm{~s})$ and the multiple scattering $\sigma_{q p}^{0}(\mathrm{~m})[2,3,20-23]$ :

$$
\sigma_{q p}^{0}=\sigma_{q p}^{0}(\mathrm{~s})+\sigma_{q p}^{0}(\mathrm{~m})
$$

The single and multiple scattering are given by

$$
\begin{gathered}
\sigma_{q p}^{0}(\mathrm{~s})=\frac{k^{2}}{2} e^{-\sigma^{2}\left(k_{s z}^{2}+k_{z}^{2}\right)} \sum_{n=1}^{\infty} \frac{\sigma^{2 n}}{n !}\left|\mathbf{I}_{q p}^{n}\right|^{2} \mathbf{S}^{(n)}\left(k_{s x}-k_{x}, k_{s y}-k_{y}\right) \\
\sigma_{q p}^{0}(\mathrm{~m})=\sum_{l=1}^{3} \sigma_{q p}^{k c_{l}}(\mathrm{~m})+\sum_{i=1}^{8} \sigma_{q p}^{c_{i}}(\mathrm{~m})+\sum_{j=9}^{14} \sigma_{q p}^{c_{j}}(\mathrm{~m})
\end{gathered}
$$

where the subscripts $p$ and $q$ denote the transmitted and received wave polarizations, respectively; $\sigma$ is the root-mean-squared (RMS) height of the rough surface; and $\mathbf{S}^{(n)}$ is the Fourier transform of the nth power of the surface correlation function $\rho$ :

$$
\mathbf{S}^{(n)}\left(K_{x}, K_{y}\right)=\iint \rho^{n}\left(\xi_{x}, \xi_{y}\right) e^{-j\left(K_{x} \xi_{x}+K_{y} \xi_{y}\right)} d \xi_{x} d \xi_{y}
$$

The roughness spectrum, $\mathbf{S}$, generally falls between Gaussian and exponential, with 1.5-power in between [2,3]. This covers most of the possible roughness spectrum for the soil surface. The factor $\mathbf{I}_{q p}^{n}$ accounting for the upward and downward scattering process under the dielectric properties of the rough surface is explicitly given in [21].

It is imperative to realize that in Equation (3), $\sigma^{2} \mathbf{S}(K, \phi)$ is the surface roughness spectrum, describing the roughness distribution over the spatial wavenumber $K$ and direction $\phi$. Such energy distribution determines the radar scattering strength through the resonance between the surface spatial wavelength and radar wavelength by which radar probes the surface. In fact, the exploring microwave does not couple with all wavelength waves, but with a specific wavenumber range of waves that have comparable roughness scales to radar wavelength, physically implying only a certain range of roughness scales that are effectively responsible for radar scattering.

Aside from the roughness spectrum, in modeling the rough surface scattering, the generic geometric parameters describing the surface are the correlation length, which measures the horizontal roughness scale $\ell$, and the RMS height $\sigma$, which is the vertical roughness scale. The ratio of RMS height to correlation length is geometrically related to the surface RMS slope. The correlation length $\ell$ is normally defined as $\rho(\ell)=e^{-1}$. In general, $\ell$ is directionally dependent for spatially anisotropic surfaces such as sea surface. For simplicity, but without loss of generality, we only discuss isotropic surface in this paper. To estimate the correlation length, it turns out that we need to know the functional form of $\rho$. For natural surfaces, the correlation length typically depends on the measured length. The variance of estimate correlation function $\hat{\rho}$ is [4]

$$
\operatorname{var}[\hat{\rho}(\zeta)]=\frac{1}{L_{s}} \int_{0}^{\infty}\left[\rho^{2}(\xi)+\rho(\xi-\zeta) \rho(\xi+\zeta)-4 \rho(\zeta) \rho(\xi) \rho(\xi+\zeta)+2 \rho^{2}(\xi) \rho^{2}(\zeta)\right] d \xi
$$

where $L_{s}$ is the profile length used to estimate $\rho$. Similarly, the variance of the estimated RMS height is

$$
\operatorname{var}(\hat{\sigma})=\frac{\sigma^{2}}{L_{s}} \int_{0}^{\infty} \rho^{2}(\xi) d \xi
$$

It is evident that both variances are strongly dependent on the shape of the correlation function, or equivalently, the roughness spectrum. The variances of both RMS height and correlation length decrease with measurement trace length. The uncertainties in RMS height and correlation length constitute (and therefore contribute uncertainty in) parameter inversion, to be treated in next section. 
To ensure confidence in the AIEM model for generating the bistatic radar data, we compared the model predictions with full-wave numerical simulations and experimental measurements, both from independent data sets. First, we used the numerical simulation of backscattering at co- and cross-polarizations by NMM3D [24-27] for an exponentially correlated surface with $\varepsilon_{r}=9.0-$ $j 2.5,15.0-j 3.5,30.0-j 4.5$ at an incident angle of $40^{\circ}$, covering $\sigma / \ell$ at the following ratios: $1 / 4$, $1 / 7,1 / 10,1 / 15$. The ratio relating to slope was from 0.021 to 0.21 . The numerical Maxwell model of three-dimensional (NMM3D) simulations was developed by Tsang et al. [24-27] to accelerate the method of moment (MoM) solutions, which efficiently combine the sparse matrix canonical grid method (SMCG), the physical-based two-grid (PBTG) method, the multi-level UV method, and the hybrid UV/PBTG/SMCG method. Further, the measured POLARSCAT data [28] for three exponentially correlated surfaces were used to validate the AIEM model. POLARSCAT is a polarimetric scatterometer operating at $\mathrm{L}, \mathrm{C}$, and $\mathrm{X}$ bands (with center frequencies of $1.25 \mathrm{GHz}, 4.75 \mathrm{GHz}$, and 9.5 $\mathrm{GHz}$, respectively) at incident angles of $10 \sim 70^{\circ}$ for $\mathrm{HH}, \mathrm{VV}$, and $\mathrm{HV} / \mathrm{VH}$ polarizations, with each surface having wet and dry conditions corresponding to different dielectric constants. The correlation length ranged from $8.4 \mathrm{~cm}$ to $9.9 \mathrm{~cm}$, while RMS height ranged from $0.4 \mathrm{~cm}$ to $1.12 \mathrm{~cm}$.

Note that the soil moisture volumetric content is related to the dielectric constant $\varepsilon_{r}[29,30]$. The comparisons of backscattering coefficients between the AIEM and NMM3D for HH, VV, and HV polarizations are shown in Figure 2a. The correlation coefficient $(r)$ between AIEM and NMM3D was greater than 0.96 , and the RMS error (RMSE) was only about $1.6 \mathrm{~dB}$ for the total of three polarizations, suggesting a good match between the model predictions and full-wave numerical simulations. The backscattering coefficients predicted by AIEM and the measured POLARSCAT are given in in Figure 2b. Overall, the correlation was very close to the 1:1 line. The correlation coefficients for both co- and cross-polarizations were about 0.9 and RMSEs were about $2.6 \mathrm{~dB}$, confirming a good match between the model prediction and the measured data over a wide range of surface and radar parameters. Among the three polarizations, the cross-polarization produced the larger root mean square error (RMSE) compared to the co-polarizations $\mathrm{HH}$ and VV. This is perhaps attributable to the higher source uncertainty in measuring cross-polarized returns, which are typically low for the backscattering of rough surfaces.

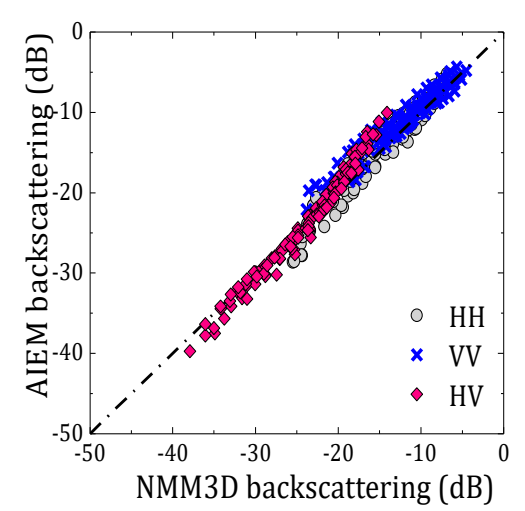

(a)

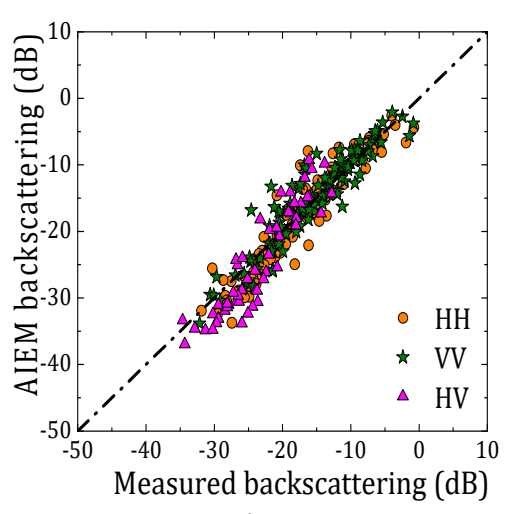

(b)

Figure 2. Correlation plot for co- (HH \& VV) and cross-polarized (HV) backscattering coefficients (a) between AIEM prediction and full-wave numerical simulations (NMM3D) and (b) between AIEM prediction and experimental measurements (POLARSCAT data).

In bistatic scattering, the AIEM prediction is validated by numerical simulations of small slope approximation (SSA) and the method of moment (MoM) for dielectric rough surfaces. As shown in Figure 3, the AIEM model predictions match quite well with the MoM simulations both in level and angular trend. Notice that strong spikes appearing in specular directions are due to the coherent scattering, which was excluded in both AIEM and SSA (small slope approximation). More comparisons 
with numerical simulations and experimental measurements presenting a higher-accuracy AIEM model in predicting bistatic scattering are given in [31].

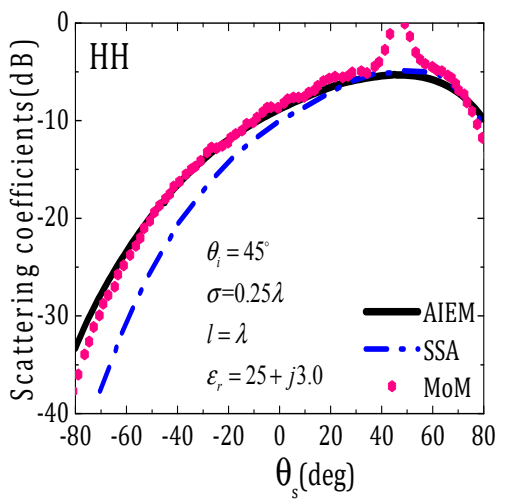

(a)

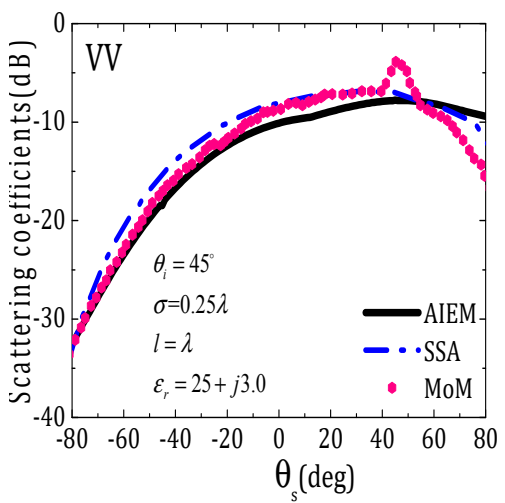

(b)

Figure 3. Comparison of bistatic scattering between AIEM prediction and numerical simulations (SSA, MoM) for a dielectric rough surface with a Gaussian-correlated function: (a) HH polarization, (b) VV polarization.

\subsection{Data Generation and Sensitivity of Bistatic Scattering to Surface Parameters}

According to the methods described in the previous section, AIEM models were validated to have a high accuracy in generating bistatic radar data. Before devising an effective approach to invert rough surface parameters, it was essential to conduct a sensitivity analysis of scattering responses to surface parameters under certain radar parameters. According to Equations (2)-(4), the three key rough surface parameters are RMS height, correlation length, and dielectric constant, which is related to moisture content. In what follows, we illustrate the bistatic scattering hemispherical plots for the dependences of these three surface parameters. As one of the key radar parameters, the incident angle is also discussed to demonstrate its effects on the scattering pattern.

Figure 4 is the hemispherical plot of polarized bistatic scattering coefficients $\mathrm{HH}, \mathrm{HV}, \mathrm{VH}$, and $\mathrm{VV}$, with an exponential correlated surface $\theta_{i}=40^{\circ}, k \sigma=1.0, m_{v}=20 \%$, showing the effect of surface roughness with normalized correlation lengths of 5 and 15. Similarly, Figure 5 shows the effect of surface roughness with a normalized RMS height of 0.5 and 1.5. The other surface parameters were fixed at $k l=5.0, m_{v}=20 \%$. The incident angle was $40^{\circ}$. In these plots, the left-hand half sphere and right-hand half sphere correspond respectively to the backward and forward scattering regions, while the horizontal axis and vertical axis represent the incident plane and cross-incident plane, respectively. From Figures 4 and 5, it can be observed that the surface roughness affects the distribution of the scattering power on the whole scattering plane. From the scattering plots in Figure 5, it is seen that there is a richer feature (in the sense of directional dependence) in the forward region than in backward region, where we see that the scattering was more omnidirectional. Equally noticeable is that in forward region generally there was a higher scattering strength, representing stronger directional dependence. Both the scattering pattern and strength in the backward region were more influenced due to the horizontal roughness $k \ell$ (when comparing the top and bottom row in Figure 5). Relatively speaking, the cross-polarizations, both $\mathrm{HV}$ and $\mathrm{VH}$, were impacted by the horizontal roughness $k \ell$ to a much lesser extent. As shown in Figure 5, as the RMS height increased, the scattering coefficients for both co-and cross-polarizations decreased near the specular region, while increasing in other directions. Note that the strong scattering of $\mathrm{HV}$ and $\mathrm{VH}$ polarizations clustered on the cross plane at a small scattering angle. The increase in $\mathrm{HH}$ polarization was more pronounced than VV polarization with increasing RMS height. In general, the dynamic range of the scattering coefficient decreased for both co- and cross-polarizations with increasing surface roughness. This phenomenon is physically understandable due to the fact that coherent scattering becomes stronger with a smaller surface roughness, and incoherent scattering is enhanced with increasing surface roughness. 

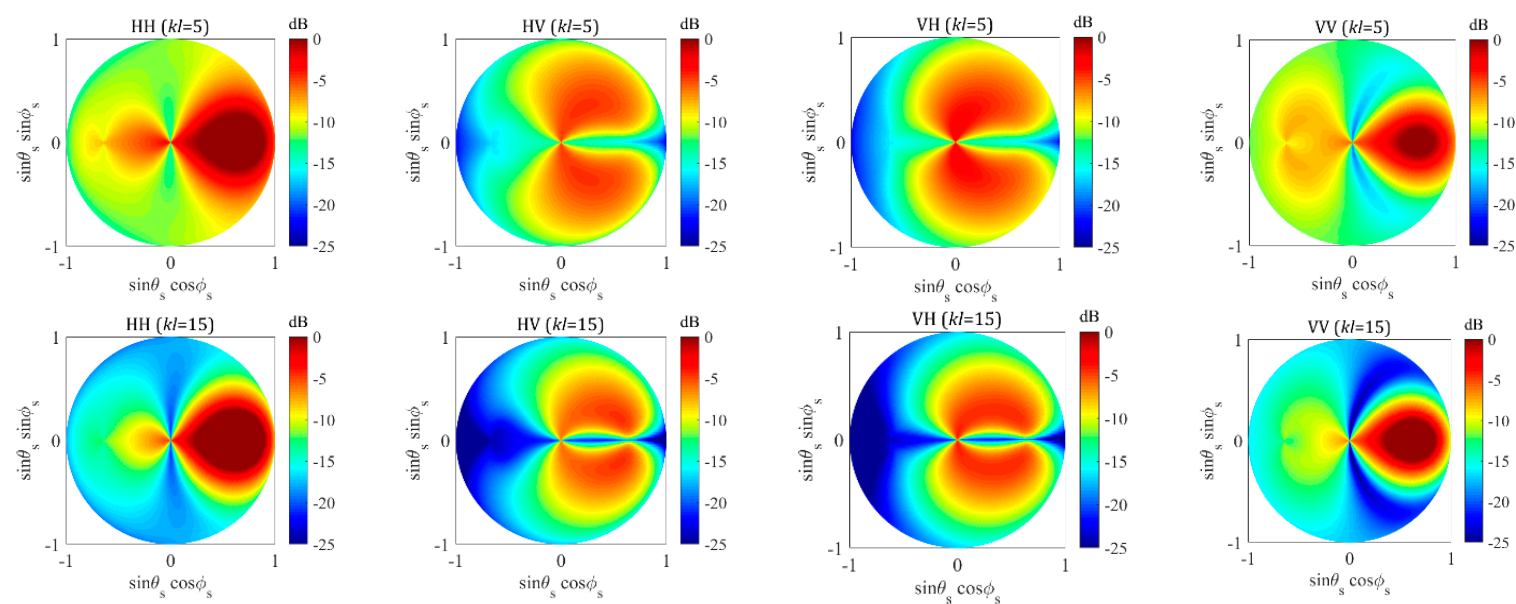

Figure 4. Hemispherical plots of polarized bistatic scattering coefficients $\mathrm{HH}, \mathrm{HV}, \mathrm{VH}$, and VV, with exponentially correlated surface $\theta_{i}=40^{\circ}, k \sigma=1.0, m_{v}=20 \%$. First row: $k \ell=5$. Bottom row: $k \ell=15$.
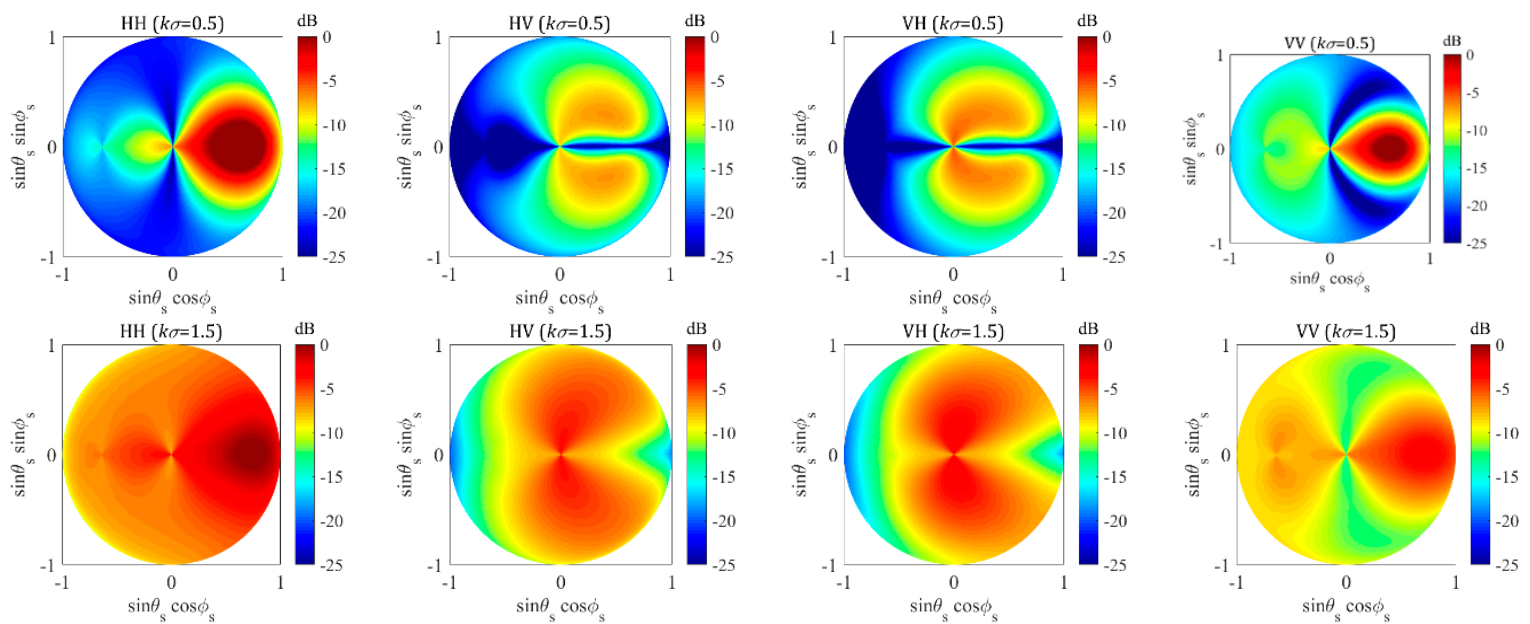

Figure 5. Hemispherical plots of polarized bistatic scattering coefficients $\mathrm{HH}, \mathrm{HV}, \mathrm{VH}$, and VV, with exponentially correlated surface $\theta_{i}=40^{\circ}, k l=5.0, m_{v}=20 \%$. Top row: $k \sigma=0.5$. Bottom row: $k \sigma=1.5$.

Similarly, the hemispherical plots of co- and cross-polarized bistatic scattering coefficients with $\theta_{i}=40^{\circ}, k \sigma=0.5, k \ell=5$, but varying the soil moisture content from $m_{v}=10 \%$ to $m_{v}=30 \%$, are illustrated in Figure 6. As is shown, with increasing moisture content the scattering strength in the forward region was greater than that in backward region for both co- and cross-polarizations. Examining Figure 6 more closely reveals that for each polarization the angular pattern remained similar as a function of moisture content. An even closer look suggests that the magnitudes for all four polarizations increased with $m_{v}$, and the scattering strength was enhanced on the whole scattering plane.

Finally, we examined the angular dependence displayed in Figure 7, which illustrates the hemispherical plots of polarized bistatic scattering coefficients $\mathrm{HH}, \mathrm{HV}, \mathrm{VH}$, and $\mathrm{VV}$, with surface parameters $k \sigma=0.5, k \ell=5, m_{v}=20 \%$ (at $\theta_{i}=20^{\circ}$ top row and $\theta_{i}=60^{\circ}$ bottom row in Figure 7). For the same set of surface parameters, the incident angle exercised a strong influence on both scattering patterns and strength for all polarizations. The strong scattering in the forward region was due to the specular scattering that moved to right hemisphere with an increase of the incident angle. At a smaller incident angle, the strength of $\mathrm{HH}$ polarization accented the forward direction with a small azimuth angular region. Yet, the region with strong scattering started to expand and disperse as the incident angle increased. In the backward region, the intensity of $\mathrm{HH}$ polarization reduced as the incident angle 
increased, but a subtle increment appeared at a large azimuth angle. A similar observation applies to the VV polarization. Meanwhile, the strength of cross-polarizations, HV and VH, quickly weakened on the whole upper hemisphere with an increasing incident angle.
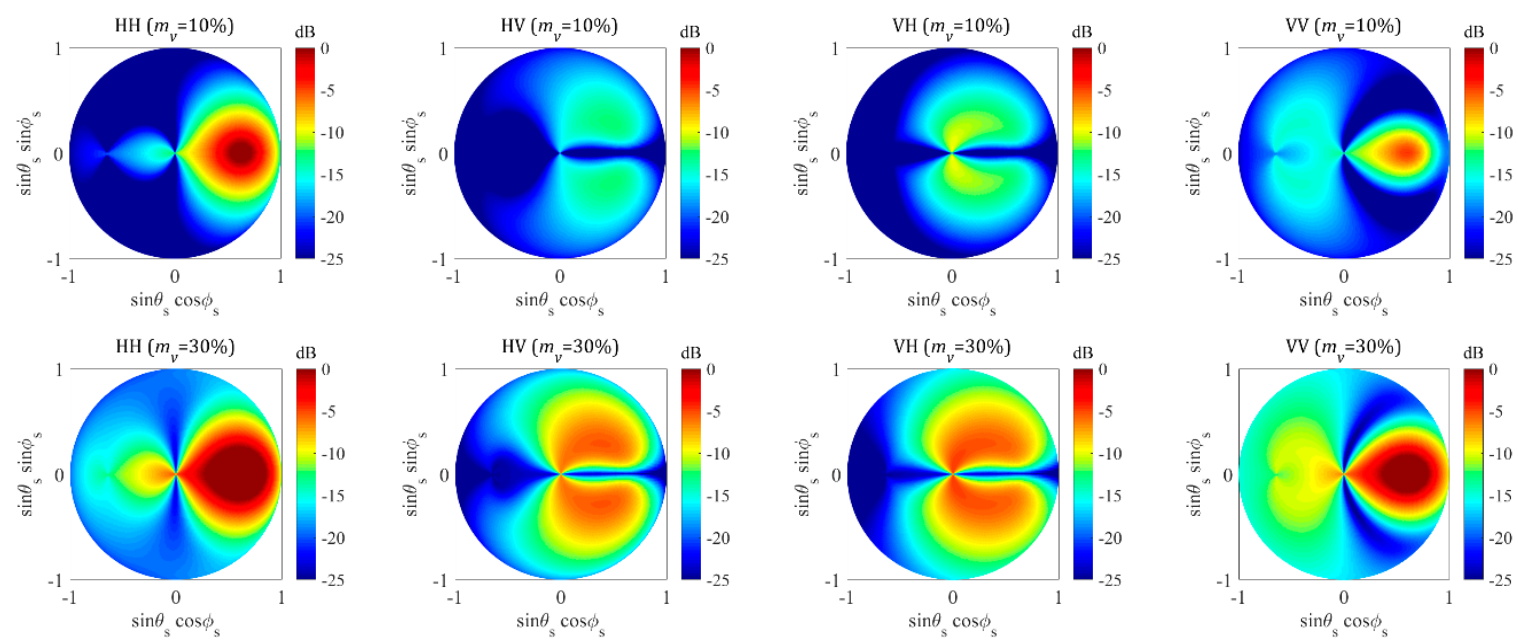

Figure 6. Hemispherical plots of polarized bistatic scattering coefficients $\mathrm{HH}, \mathrm{HV}, \mathrm{VH}$, and VV, with exponentially correlated surface $\theta_{i}=40^{\circ}, k \sigma=0.5, k \ell=5$. Top row: $m_{v}=10 \%$. Bottom row: $m_{v}=30 \%$
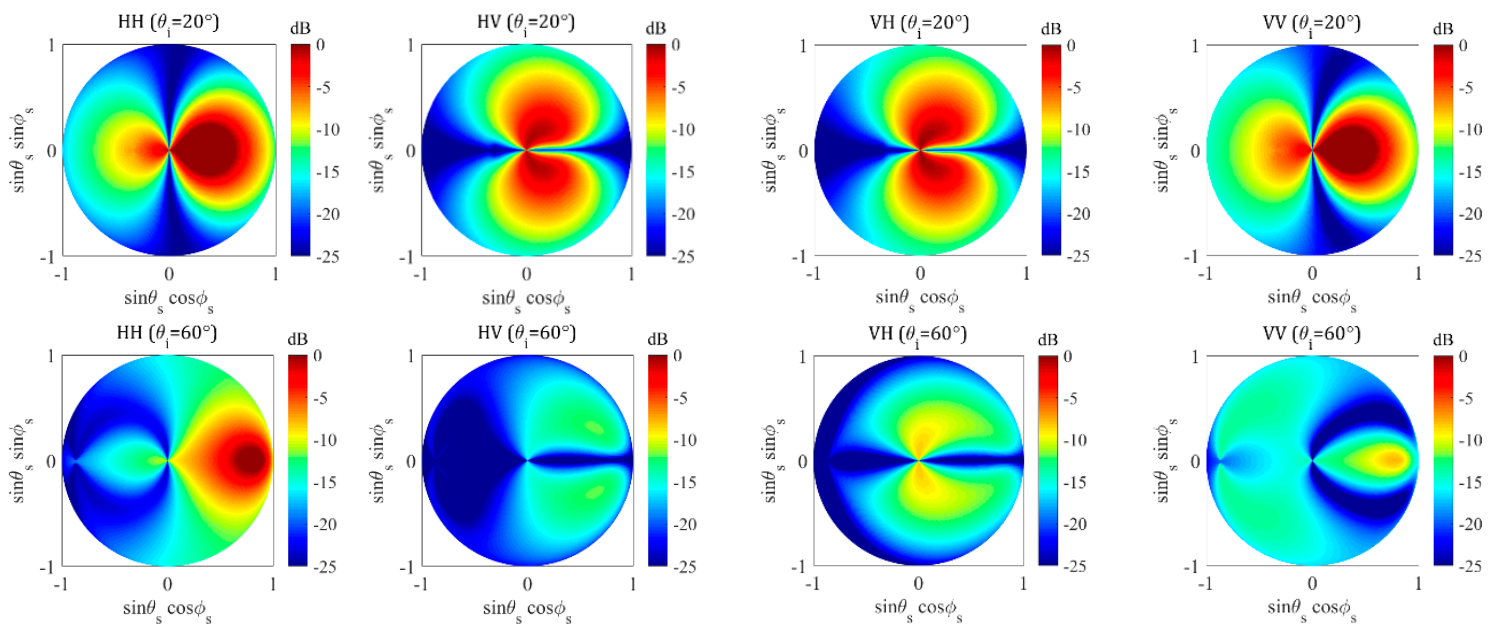

Figure 7. Hemispherical plots of polarized bistatic scattering coefficients $\mathrm{HH}, \mathrm{HV}, \mathrm{VH}$, and VV, with exponentially correlated surface $k \sigma=0.5, k \ell=5, m_{v}=20 \%$. First row: $\theta_{i}=20^{\circ}$. Bottom row: $\theta_{i}=60^{\circ}$.

From the scattering plots of Figures 5-7, the dependence of surface parameters and radar parameters, in which only selected ones were chosen for illustration, are persistently and strongly coupled. This makes surface parameter retrieval from bistatic scattering, both in terms of pattern and strength, extremely complicated, although the features are much richer. To this end, feature selection by proper data models and mapping from feature space to parameter space are essential and schemes are developed in the following section.

\section{Surface Parameters Retrieval}

\subsection{A Neural Network Approach}

In the previous section, the AIEM model was validated and the sensitivity of the bistatic scattering responding to surface parameters and radar parameters was analyzed. To better invert the surface parameters of interest, a proper data model is essential to enable a mapping of the measurement 
domain to the feature domain that is of interest. We detail how we came up with a good scheme to improve retrieval accuracy and efficiency. The radar scattering from a rough surface can be modeled as

$$
\mathbf{b}=\mathbf{W} \mathbf{x}+\mathbf{u}
$$

where $\mathbf{x}$ is the surface parameters vector; matrix $\mathbf{W}$ relates the surface parameters vector $\mathbf{x}$ to radar scattering coefficients $\mathbf{b}$; and $\mathbf{u}$ represents the measurement error vector induced by system and calibration errors, and speckle noise, among other factors. In a statistical sense, $\mathbf{x}$ constitutes a random variable due to spatially and temporally varying properties, such that

$$
\mathbf{x}=\mathbf{x}_{t}+\mathbf{x}_{n}
$$

where $\mathbf{x}_{t}$ is the true variable, and $\mathbf{x}_{n}$ is the noise term.

In practice, the "truth" is never obtainable; it is always vague. Statistically, $\mathbf{x}_{t}$ and $\mathbf{x}_{n}$ may be assumed to be, as they usually are, uncorrelated, such that $\mathbf{x}$ is an unbiased estimate of $\mathbf{x}_{t}$, i.e.,

$$
E(\mathbf{x})=E\left(\mathbf{x}_{t}\right), \forall \mathbf{x}_{n} \sim N\left(0, \sigma_{\mathbf{x}_{n}}^{2}\right)
$$

where $E$ denotes the statistical mean, and $\sigma_{\mathbf{x}_{n}}^{2}$ is a variance of $\mathbf{x}_{n}$.

Note that the radar response is formed by, in general, the scattering matrix. For the purpose of this paper and, without loss of generality, we assume it is formed by multi-polarized scattering coefficients:

$$
\mathbf{b}=\left[\sigma_{h h}^{o}, \sigma_{h v}^{o}, \sigma_{v h}^{o} \sigma_{v v}^{o}\right]^{\mathrm{t}}
$$

where each component may contain multi-angular observables. The rough surface parameters of interest are the RMS height, correlation length, and moisture content, denoted by the $\mathbf{x}$ vector:

$$
\mathbf{x}=\left[\sigma, \ell, m_{v}\right]^{\mathrm{t}}
$$

or more conveniently, normalized to the wavenumber:

$$
\mathbf{x}=\left[k \sigma, k \ell, m_{v}\right]^{\mathrm{t}}
$$

It has been argued that [32] non-quadratic regularization is practically effective in minimizing the clutter while emphasizing the target features via

$$
\hat{\mathbf{x}}=\operatorname{argmin}\left\{\|\mathbf{b}-\mathbf{W x}\|_{2}^{2}+\gamma^{2}\|\mathbf{x}\|_{p}^{p}\right\}
$$

where \|\|$_{p}$ denotes $\ell_{p}-\operatorname{norm}(p \leq 1), \gamma^{2}$ is a scalar parameter, and $\left\{\|\mathbf{b}-\mathbf{W x}\|_{2}^{2}+\gamma^{2}\|\mathbf{x}\|_{p}^{p}\right\}$ is recognized as the cost or objective function.

Direct solving of Equation (8) is perhaps possible, but demands intensive computational resources. From the preceding section, we also see that the scattering behavior, both pattern and strength, is complicatedly determined, in a stochastic sense, by three surface parameters. Hence, in search for the cost function minima in Equation (8), we may seek a neural network approach. Perhaps one disadvantage of the neural network is that it constitutes a black box for most users. Extensive studies show that it is a powerful tool for handling complex problems involving bulky volume data in high dimensional feature space. By inverting parameter vectors from measurements, a neural network offers an effective and efficient approach, and will be detailed in the following section.

Modified from multi-layer perceptron (MLP), a dynamic learning neural network (DLNN) was proposed [33] and is adopted in this paper. Figure 8 schematically depicts the configuration of a dynamic learning neural network (DLNN). It features every node at an input layer, and all hidden layers fully connected to the output layer. The activation function is removed from each output node, 
and the output of the modified network can be characterized as the weighted sum of the polynomial basis vectors. Such modifications form a condensed model of the MLP in which the output is a weighted sum of the compositions of polynomials. Hence, with measurement error matrix $\mathbf{u}$, as in Equation (8), $\mathbf{W}$ is the network weight matrix.

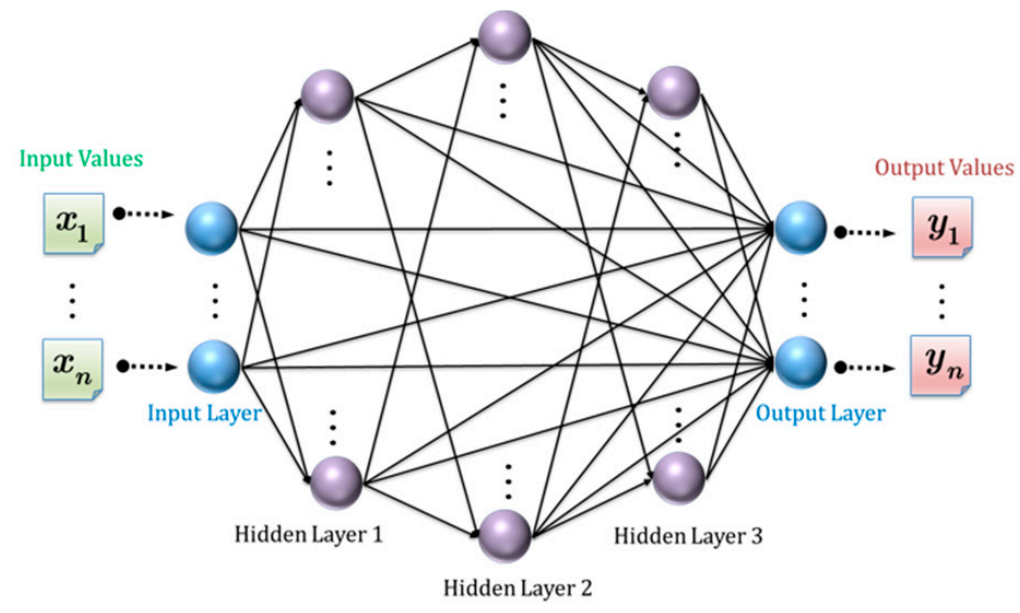

Figure 8. Network structure of a dynamic learning neural network (DLNN). It features every node at an input layer, and all hidden layers fully connected to the output layer; the activation function is removed from each output node, and the output of the modified network can be characterized as the weighted sum of the polynomial basis vectors.

\subsection{Training Scheme}

The network training or learning scheme, based on the Kalman filter technique [34] that lends itself to a highly dynamic and adaptive merit during the learning stage, is described below. To begin, the basic concept of Kalman filtering is briefly described, with notation given in Figure 9.

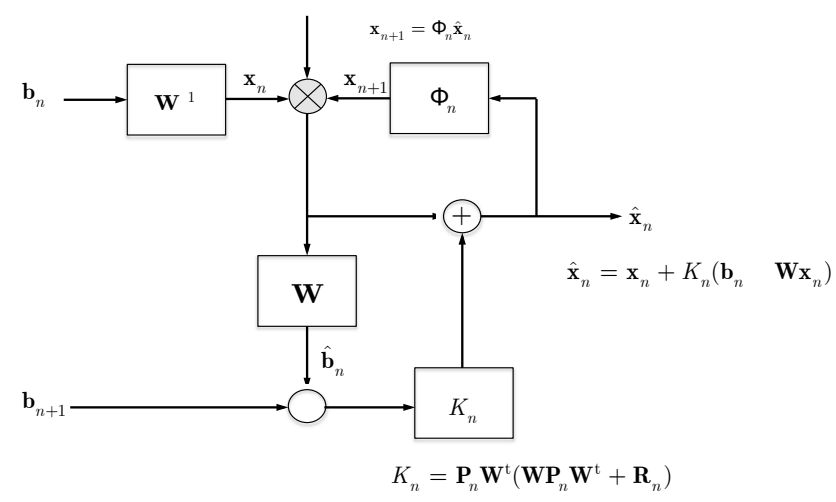

Figure 9. Schematic description of Kalman filtering.

The measurement equation takes the form for the one-step n:

$$
\mathbf{b}_{n}=\mathbf{W} \mathbf{x}_{n}+\mathbf{u}_{n}
$$

where the subscript $n$ denotes the measurement at a discrete $n$th time step. The process equation relates the transitionary states of the surface-parameters vector $\mathrm{x}$ :

$$
\mathbf{x}_{n+1}=\Phi_{n} \mathbf{x}_{n}+\mathbf{B}_{n} \mathbf{v}_{n}
$$

where $\Phi_{n}$ is a transition matrix, $\mathbf{v}_{n}$ is the process error vector, and $\mathbf{B}_{n}$ is the error matrix which, in our case, is a diagonal matrix. 
The measurement error and process error can be assumed to be statistically independent, i.e.,

$$
\begin{gathered}
\mathrm{E}\left[\mathbf{u}_{m} \mathbf{v}_{n}^{\mathrm{t}}\right]=0, \quad \forall m, n . \\
\hat{\mathbf{x}}_{n}=\widetilde{\mathbf{x}}_{n}+K_{n}\left(\mathbf{b}_{n}-\mathbf{W} \widetilde{\mathbf{x}}_{n}\right) \\
\widetilde{\mathbf{x}}_{n+1}=\Phi_{n} \hat{\mathbf{x}}_{n}
\end{gathered}
$$

where $\widetilde{\mathbf{x}}_{n}$ is the one-step predicted estimate, $\hat{\mathbf{x}}_{n}$ is the filter estimate of the desired $\mathbf{x}_{n}$, and $K_{n}$ is the computed Kalman gain. For numerical stability, its computation takes the following steps:

$$
\begin{gathered}
K_{n}=\widetilde{\mathbf{P}}_{n} \mathbf{W}^{\mathrm{t}}\left(\mathbf{W} \widetilde{\mathbf{P}}_{n} \mathbf{W}^{\mathrm{t}}+\mathbf{R}_{n}\right) \\
\hat{\mathbf{P}}_{n}=\widetilde{\mathbf{P}}_{n}-K_{n}\left(\widetilde{\mathbf{P}}_{n} \mathbf{W}^{\mathrm{t}}\right)^{\mathrm{t}} \\
\widetilde{\mathbf{P}}_{n+1}=\Phi_{n} \hat{\mathbf{P}}_{n} \Phi_{n}^{\mathrm{t}}+\mathbf{B}_{n} \mathbf{Q}_{n} \mathbf{B}_{n}^{\mathrm{t}}
\end{gathered}
$$

where $\widetilde{\mathbf{P}}_{n}, \hat{\mathbf{P}}_{n}$ are the one-step predicted and filter estimate error covariance matrices, respectively. The initial state can be set as $\widetilde{\mathbf{P}}_{1}=\mathrm{E}\left[\mathbf{x}_{1} \mathbf{x}_{1}^{t}\right]$.

For the error vectors, it is physically reasonable to assume that for both measurement and process error vectors

$$
\begin{aligned}
& \mathrm{E}\left[\mathbf{u}_{m} \mathbf{u}_{n}^{\mathrm{t}}\right]=\left\{\begin{array}{c}
\mathbf{R}_{n}, m=n \\
0, m \neq n
\end{array}\right. \\
& \mathrm{E}\left[\mathbf{v}_{m} \mathbf{v}_{n}^{\mathrm{t}}\right]=\left\{\begin{array}{c}
\mathbf{Q}_{n}, m=n \\
0, m \neq n
\end{array}\right.
\end{aligned}
$$

where $\mathbf{R}_{n}, \mathbf{Q}_{n}$ are error covariance matrices, respectively. The process error and the measurement error in radar observation may be reasonably assumed to be statistically independent and can be modeled as zero mean, white noise process

From our modified MLP structure, each updated estimate of the neural network weight is computed from the previous estimate and the new input data. The weights connected to each output node can be updated independently such that the vector problem can therefore be decomposed into $L$ scalar problems as

$$
b_{\kappa}=\mathbf{w}_{\kappa} \mathbf{x} \quad(\kappa=1,2, \cdots, L)
$$

Applying the Kalman filtering technique schematically described in Figure 9, Equation (25) can be modeled as

$$
\begin{gathered}
b_{\kappa}^{i}=\mathbf{w}_{\kappa}^{i} \mathbf{x}+v_{\kappa}^{i} \\
\mathbf{w}_{\kappa}^{i+1}=\mathbf{w}_{\kappa}^{i} \mathbf{A}^{i}+u_{\kappa}^{i} \mathbf{B}^{i}
\end{gathered}
$$

where the superscript $i$ denotes the $i^{\text {th }}$ training pattern with the total of $N$; $\mathbf{A}^{i}$ is a $M$ by $M$ state transition matrix; $\mathbf{B}^{i}$ is a $M$ by $M$ diagonal matrix; $\boldsymbol{u}_{\kappa}^{i}$ represents a 1 by $M$ process error vector, with $M$ the dimension of concatenated activations in modified MLP; and $v_{\kappa}^{i}$ is a scalar measurement error.

The update of network weights is according to the following recursions:

$$
\begin{gathered}
\hat{\mathbf{w}}_{\kappa}^{i}=\widetilde{\mathbf{w}}_{\kappa}^{i}+\mathbf{g}_{\kappa}^{i}\left[d_{\mathcal{K}}^{i}-\widetilde{\mathbf{w}}_{\kappa}^{i} \mathbf{x}\right] \quad(i=1,2, \cdots, N) \\
\widetilde{\mathbf{w}}_{\kappa}^{i+1}=\hat{\mathbf{w}}_{\kappa}^{i} \mathbf{A}^{i}
\end{gathered}
$$

where $d_{\kappa}^{i}$ is the desired output, $\widetilde{\mathbf{w}}_{\kappa}^{i}$ is the one-step predicted estimate, and $\hat{\mathbf{w}}_{\kappa}^{i}$ is the filter estimate of $\mathbf{w}_{\kappa}^{i}$, with $\mathbf{g}_{\kappa}^{i}$ the computed Kalman gain, which is viewed as an adaptive learning rate and is computed according to Equations (20)-(24). The MLP together with learning by the Kalman filter is expected to resolve the highly nonlinear, complex decision boundary problems, as demonstrated in the following section. 


\section{Results and Discussions}

\subsection{Data Input-Output and Training Samples}

In this study, two network configurations were devised, with three input parameters for backscattering and four input parameters for bistatic scattering (see Table 1). The outputs of the network were normalized surface roughness (RMS height $k \sigma$, correlation length $k \ell$ ), and soil moisture $m_{v}$. Three roughness spectra-Gaussian, exponential, and 1.5-power-were all included in the simulation. The inputs are given below.

Table 1. Input-output of a dynamic learning neural network (DLNN).

\begin{tabular}{|c|c|c|c|}
\hline \multicolumn{2}{|c|}{$\begin{array}{l}\text { Backscattering } \\
\text { (Three-Input) }\end{array}$} & \multicolumn{2}{|c|}{$\begin{array}{c}\text { Bistatic Scattering } \\
\text { (Three-Input \& Four-Input) }\end{array}$} \\
\hline Input & Output & Input & Output \\
\hline $\begin{array}{c}\mathrm{HH}, \mathrm{VV} \text { and } \mathrm{HV} \\
\text { polarized backscattering } \\
\text { coefficients of } \\
\mathbf{b}=\left[\sigma_{h h^{\prime}}^{o} \sigma_{h v}^{o}, \sigma_{v v}^{o}\right]^{\mathrm{t}}\end{array}$ & $\begin{array}{c}\text { Normalized surface } \\
\text { roughness and soil } \\
\text { moisture } \\
\mathbf{x}=\left[k \sigma, k \ell, m_{v}\right]^{\mathrm{t}}\end{array}$ & $\begin{array}{c}\mathrm{HH}, \mathrm{HV}, \mathrm{VH} \text { and VV } \\
\text { polarized bistatic } \\
\text { coefficients } \\
\mathbf{b}=\left[\sigma_{h h^{\prime}}^{o}, \sigma_{h v^{\prime}}^{o}, \sigma_{v v}^{o}\right]^{\mathrm{t}} \\
\mathbf{b}=\left[\sigma_{h h^{\prime}}^{o} \sigma_{h v^{\prime}}^{o} \sigma_{v h}^{o} \sigma_{v v}^{o}\right]^{\mathrm{t}}\end{array}$ & $\begin{array}{c}\text { Normalized surface } \\
\text { roughness and soil } \\
\text { moisture } \\
\mathbf{x}=\left[k \sigma, k \ell, m_{v}\right]^{\mathrm{t}}\end{array}$ \\
\hline
\end{tabular}

Backscattering: In this case, $\mathrm{HH}-, \mathrm{VV}-$, and $\mathrm{HV}(=\mathrm{VH})$-polarized backscattering coefficients were simultaneously fed into the dynamic learning neural network. The incident angles were set at $10 \sim 60^{\circ}$.

Bistatic Scattering: In this setup, inputs chosen from $\mathrm{HH}-, \mathrm{HV}-, \mathrm{VH}-$, and VV-polarized bistatic scattering coefficients at different incident angles, scattering angles, and scattering azimuthal angle were fed into the network. Both the incident angles and scattering angle were selected at $10 \sim 60^{\circ}$. The scattering azimuthal angle was set for the forward region at $0 \sim 90^{\circ}$ and for the backward region at $90 \sim 180^{\circ}$. For bistatic scattering, we devised three inputs (as in backscattering) for comparison of inversion performance under the same number of inputs.

It follows that the network configuration, after trial and error, was given with a predetermined threshold of $0.1 \%$ (Table 2). A total of 10,450 data sets were selected randomly as training data from the 15,014 data sets for backscattering, with 4564 data sets used as testing data. As a rule of thumb, $70 \%$ of the total data sets were used for training (i.e., 127,240 data sets are chosen randomly as training data from the 181,770 data sets for bistatic scattering), and 54,530 data sets were used as testing data, from which the backward region and forward region cases each accounted for half of the data. The data sets are randomly selected from the database simulated by the AIEM model with the ranges of the surface and radar parameters listed in Table 3. The step size of the discretized scattering azimuthal angle was set to $10^{\circ}$, and the discretized incident and scattering angles were both set to $1^{\circ}$.

Table 2. DLNN configurations for three-input and four-input cases.

\begin{tabular}{ccc}
\hline Configuration & Three-Input & Four-Input \\
\hline Nodes of input layer & 3 & 4 \\
Nodes of output layer & 3 & 3 \\
Hidden layer & 2 & 2 \\
Nodes of each hidden layer & 100 & 100 \\
Threshold & $0.1 \%$ & $0.1 \%$ \\
\hline
\end{tabular}

The network training was accomplished by mapping input-output pairs that were randomly selected from the database simulated by the AIEM model with the range of surface and radar parameters listed in Table 3. As rule of thumb, $70 \%$ of the data was selected, randomly, as the training set, with the rest as the testing set. 
Table 3. Radar parameters for generating training samples.

\begin{tabular}{ccc}
\hline Parameters & Backscattering & Bistatic Scattering \\
\hline$k \sigma$ & $0.1 \sim 0.8$ & $0.1 \sim 0.8$ \\
$k \ell$ & $1 \sim 7$ & $1 \sim 7$ \\
$m_{v}$ & $0 \sim 0.5$ & $0 \sim 0.5$ \\
$\theta_{i}$ & $10^{\circ} \sim 60^{\circ}$ & $10^{\circ} \sim 60^{\circ}$ \\
$\theta_{S}$ & $=\theta_{i}$ & $10^{\circ} \sim 60^{\circ}$ \\
$\phi_{S}$ & $180^{\circ}$ & $0^{\circ} \sim 90^{\circ}$ (forward) \\
$k \sigma / k \ell$ & $0.1 \sim 0.4$ & $90^{\circ} \sim 180^{\circ}$ (backward) \\
\hline $\mathrm{S}$ & \multicolumn{2}{c}{ Gaussian, Exponential, 1.5 -Power } \\
\hline
\end{tabular}

\subsection{Retrieval Results}

After completing the training, the DLNN entered the process stage. By randomly selecting $30 \%$ testing sample, the surface parameter retrieval was performed via the DLNN. This was indeed a highly nonlinear mapping of feature sets (by training) onto the surface domain (by process). The retrieval performance between the network-inverted result and the model-observed data, using backscattering data only (i.e., three-input), can be seen in Figure 10. For three surface parameters of interest, the normalized root-mean-squared errors (nRMSE) were 0.074, 0.075 and 0.070 for RMS height, correlation length, and soil moisture, respectively, and correlation coefficients were larger than 0.95, which was quite satisfactory; among the three parameters, the inversion of $k \ell$ was poorer. The reason for this is that, as discussed previously, the estimation of correlation length always poses higher errors due to a higher uncertainty of the functional form of the correlation function. Several samples of inversion results between the measured data (POLARSCAT) and the network inversion are presented in Table 4 . As we can see, the retrieval performance of these three parameters can achieve a satisfactory accuracy.
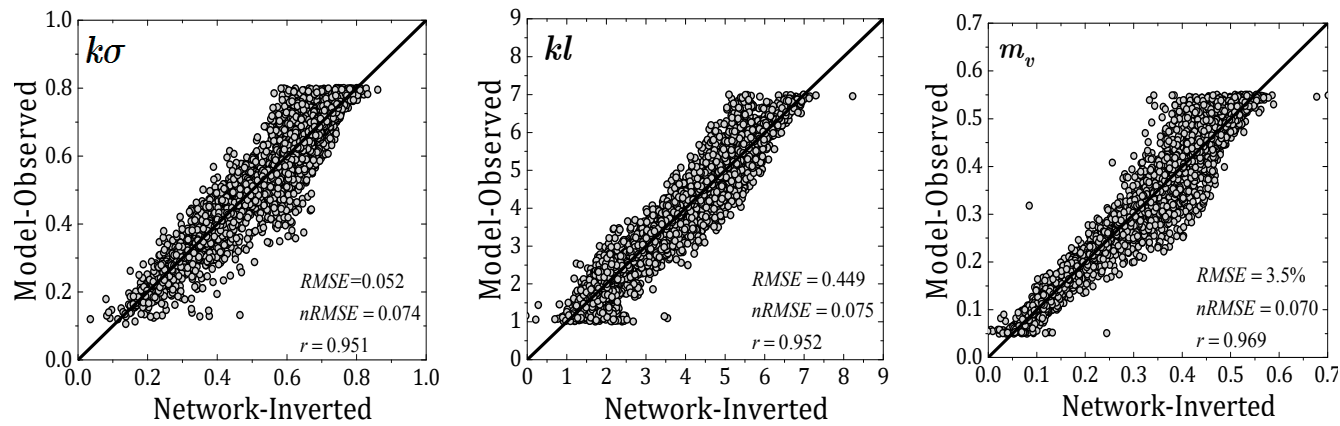

Figure 10. Retrieval of surface roughness and soil moisture from backscattering data by three inputs-three outputs DLNN configuration. Both root-mean-squared errors (RMSE) and normalized root-mean-squared errors (nRMSE) for each case are shown.

When it comes to inversion from bistatic scattering data, we can examine the performance of using forward, backward, and full (backward plus forward), respectively. This might be practically useful since, in setting up the bistatic observation, the transmitter and receiver may be in specular or off-specular geometries. The correlation between the network-inverted result and model-observed data is shown in Figure 11. The inversion results were relatively close to the 1:1 line, indicating a quite-favorable correlation between the inversion and the truth data. It is worth noting that retrieval from bistatic scattering data performed better than from backscattering, especially for soil moisture inversion. Interestingly, the inversion results performed better at the forward region than at the backward region. This can also be seen quantitatively in Table 5, from which we can read the nRMSE being $0.060,0.088$, and 0.044 in the backward region and $0.045,0.071$, and 0.018 in the forward region for RMS height, correlation length, and soil moisture, respectively. The overall nRMSE and correlation 
coefficient $(r)$ for backscattering and bistatic scatter are also given in Table 5. Typically, among the three surface parameters being inverted, the soil moisture tended to experience the least error, regardless of retrieval from backscattering data or bistatic scattering data. Better inversion performance seems to have been gained by use of scattering data from the forward region. Based on this point and compared to the backscattering, it is not surprising that if we combine the scattering data from the forward and backward regions, the best retrieval accuracy in terms of nRMSE and the correlation coefficient can be obtained. This is also evident from Table 5, where the smaller nRMSE and higher correlation coefficient can be read as "Full" when compared to those for backscattering. It is apparently a conviction that the dynamic learning neural network as presented is able to tackle a bulky volume of data, either in training stage or operational stage, and thus achieve superior retrieval accuracy.

Table 4. Surface parameters between measured data (POLARSCAT) and DLNN inversion.

\begin{tabular}{|c|c|c|c|c|c|}
\hline \multicolumn{3}{|c|}{$\begin{array}{c}\text { Truth } \\
\text { (POLARSCAT Data) }\end{array}$} & \multicolumn{3}{|c|}{$\begin{array}{c}\text { Inverted } \\
\text { (Network-Inverted) }\end{array}$} \\
\hline$k \sigma$ & $k \ell$ & $m_{v}$ & $k \sigma$ & $k \ell$ & $m_{v}$ \\
\hline 0.949 & 2.768 & 0.142 & 0.907804 & 2.75213 & 0.141224 \\
\hline 3.004 & 8.765 & 0.172 & 7.1636 & 12.1984 & 0.232222 \\
\hline 0.352 & 2.617 & 0.266 & 0.346407 & 2.61012 & 0.265967 \\
\hline 0.126 & 2.62 & 0.126 & 0.126175 & 2.62442 & 0.125997 \\
\hline 0.352 & 2.617 & 0.14 & 0.371045 & 2.6396 & 0.14006 \\
\hline 0.101 & 3.098 & 0.09 & 0.103238 & 3.15896 & 0.08993 \\
\hline 1.114 & 8.287 & 0.14 & 1.20734 & 8.85712 & 0.137385 \\
\hline 0.796 & 16.594 & 0.253 & 1.66042 & 5.38294 & 0.364186 \\
\hline 3.004 & 8.765 & 0.172 & 3.03834 & 8.83309 & 0.172412 \\
\hline 0.949 & 2.768 & 0.172 & 0.948958 & 2.76862 & 0.172035 \\
\hline 6.009 & 17.529 & 0.172 & 2.69795 & 5.08291 & 0.16386 \\
\hline 6.009 & 17.529 & 0.142 & 5.91543 & 17.6258 & 0.142792 \\
\hline 0.352 & 2.617 & 0.266 & 0.351636 & 2.61618 & 0.266002 \\
\hline 2.228 & 16.574 & 0.14 & 2.22803 & 16.5737 & 0.140001 \\
\hline 0.796 & 16.594 & 0.253 & 0.797609 & 16.6051 & 0.252946 \\
\hline 2.228 & 16.574 & 0.14 & 2.24075 & 16.5635 & 0.139998 \\
\hline 0.398 & 8.297 & 0.253 & 0.396886 & 8.30057 & 0.252988 \\
\hline 3.004 & 8.765 & 0.172 & 3.06484 & 8.54212 & 0.172106 \\
\hline Error & \multicolumn{2}{|c|}{$k \sigma$} & \multicolumn{2}{|c|}{$k \ell$} & $m_{v}$ \\
\hline RMSE & \multicolumn{2}{|c|}{1.2701} & \multicolumn{2}{|c|}{4.0330} & $2.987 \%$ \\
\hline nRMSE & \multicolumn{2}{|c|}{0.2149} & \multicolumn{2}{|c|}{0.2705} & 0.2134 \\
\hline$r$ & \multicolumn{2}{|c|}{0.7706} & \multicolumn{2}{|c|}{0.7761} & 0.9150 \\
\hline
\end{tabular}
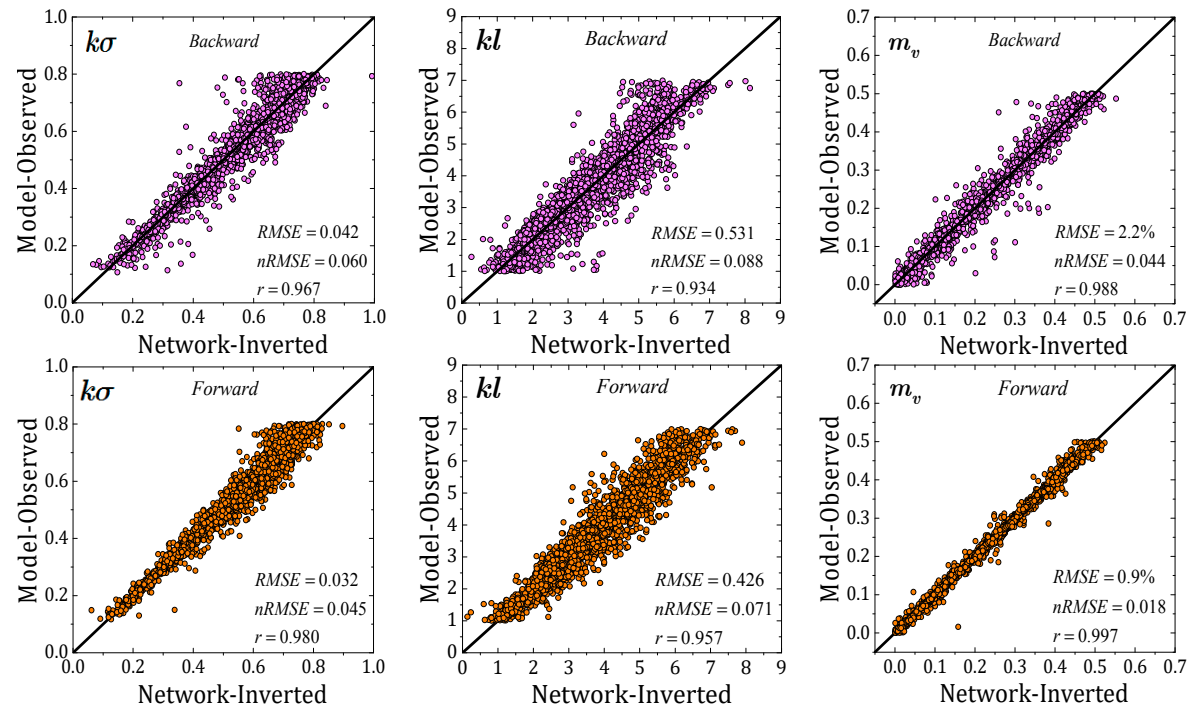

Figure 11. Retrieval performance of surface roughness and soil moisture from bistatic scattering data (four-input). Top row: backward region. Bottom row: forward region. 
Table 5. DLNN inversion performance using backscattering and bistatic scattering.

\begin{tabular}{|c|c|c|c|c|c|}
\hline \multicolumn{2}{|c|}{ Backscattering \& Bistatic Scatter } & Error & $k \sigma$ & $k \ell$ & $m_{v}$ \\
\hline \multirow{2}{*}{\multicolumn{2}{|c|}{$\begin{array}{l}\text { Backscattering } \\
\text { (Three-input) }\end{array}$}} & nRMSE & 0.074 & 0.075 & 0.070 \\
\hline & & $\mathrm{r}$ & 0.951 & 0.952 & 0.969 \\
\hline \multirow{4}{*}{ (Three-input) } & \multirow{2}{*}{ Backward } & nRMSE & 0.075 & 0.087 & 0.039 \\
\hline & & $\mathrm{r}$ & 0.951 & 0.936 & 0.990 \\
\hline & \multirow{2}{*}{ Forward } & nRMSE & 0.040 & 0.070 & 0.044 \\
\hline & & $\mathrm{r}$ & 0.985 & 0.959 & 0.988 \\
\hline & \multirow{2}{*}{$\begin{array}{c}\text { Full } \\
\text { (Backward + Forward) }\end{array}$} & nRMSE & 0.058 & 0.097 & 0.042 \\
\hline & & $\mathrm{r}$ & 0.970 & 0.949 & 0.991 \\
\hline \multirow{6}{*}{$\begin{array}{c}\text { Bistatic } \\
\text { scattering } \\
\text { (Four-input) }\end{array}$} & \multirow{2}{*}{ Backward } & nRMSE & 0.060 & 0.088 & 0.044 \\
\hline & & $\mathrm{r}$ & 0.967 & 0.934 & 0.988 \\
\hline & \multirow{2}{*}{ Forward } & nRMSE & 0.045 & 0.071 & 0.018 \\
\hline & & $\mathrm{r}$ & 0.980 & 0.957 & 0.997 \\
\hline & Full & nRMSE & 0.053 & 0.080 & 0.034 \\
\hline & (Backward + Forward) & $\mathrm{r}$ & 0.974 & 0.945 & 0.992 \\
\hline
\end{tabular}

There is a much higher degree of freedom to test the inversion from bistatic scattering compared to backscattering, as well as to choose the inputs, depending on the physical feasibility of the measurements. We can have measurements at the backward, forward, or combined forward and backward regions to input to the DLNN. In terms of polarization, we can have four polarizations in bistatic - two co-polarizations, two cross-polarizations, two-polarizations, or one cross-polarization. Figure 12 shows the retrieval performance of surface roughness and soil moisture from bistatic scattering data (three-input). The simulation test confirms the inversion performance of the neural network in terms of training and operation. From Table 5, we can see that the retrieval accuracy is higher using forward bistatic data than backward. The impact of polarization for bistatic cases seems not as significant as that for backscattering cases. More importantly, for bistatic case, three inputs do not necessarily produce higher retrieval accuracy than four inputs do. It is the number of features that determines the training effectiveness and thus the retrieval accuracy. It becomes clear at this point that under the same number of inputs, inversion accuracy is higher from bistatic scattering than from backscattering. This is likely due to the fact that the bistatic scattering measurements can better separate the coupling effect of roughness and dielectric constant. For this, the sensitivity analysis given in Figures $4-7$ is critical for feature selection to train the neural network.
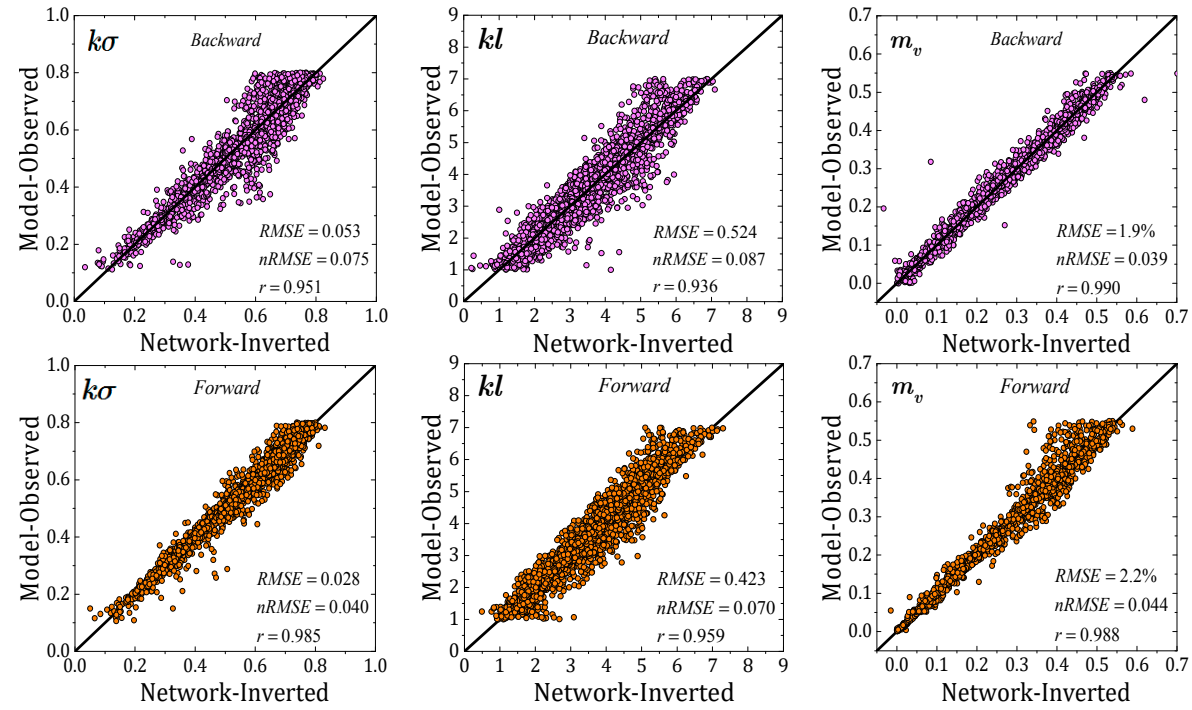

Figure 12. Retrieval performance of surface roughness and soil moisture from bistatic scattering data (three-input). Top row: backward region. Bottom row: forward region. 
At this point, it is of relevance to demonstrate the retrieval of soil surface parameters from bistatic radar measurements [35-38], including roughness and moisture content, using the model-trained DLNN. A bistatic measurement facility (BMF) [35,36] was designed and constructed consisting of a 10 and $35 \mathrm{GHz}$ polarimetric radar system for the purpose of characterizing the 3D bistatic scattering response of rough dielectric surfaces. According to [35], a measurement with $10 \mathrm{GHz}$ from a Gaussian random rough surface, with a normalized RMS height of $k \sigma=0.2$ and a normalized correlation length of $k l=1.0$, was water-soaked bricks in which the real part of the dielectric constant was 62. The incident angle and scattering angle were both set as $45^{\circ}$, and the scattering azimuth angle was changed from $180^{\circ}$ to $-180^{\circ}$. [36] gives the measurements of a rough soil surface with $35 \mathrm{GHz}$ using an indoor bistatic measurement facility (IBMF). At $35 \mathrm{GHz}$, the surface was assumed to be an exponentially correlated function with the normalized the RMS height and correlation length being 3.28 and 29.6, respectively. The effective soil permittivity was 3.5-j0.05. The measured bistatic scattering coefficient was a function of the scattering angle $\left(0 \sim 70^{\circ}\right)$ in the backward direction $\left(\phi_{s}=180^{\circ}\right)$ and forward direction $\left(\phi_{s}=0^{\circ}\right)$, and the incident angle was $20^{\circ}$. Another data set is taken from [37,38], where the bistatic rough dielectric surface scattering was measured at the European Microwave Signature Laboratory (EMSL) at $\left(-10 \sim-50^{\circ}\right)$ of incidence and scattering angles with 1.5-18.5 GHz of frequency. The surface was characterized by a Gaussian correlation function with $\sigma=2.5 \mathrm{~cm}, l=6 \mathrm{~cm}$ of surface roughness.

To assess the inversion performance and be more quantitative, we list the numeric results in Table 6, showing the measured $k \sigma, k \ell$, and $m_{v}$, along with the inverted data. Of the eight total test sets, the inverted results are in good agreement with the measured data. Relatively, the difference between the measured and inverted data are well within 20\% error. The RMSE, nRMSE, and correlation coefficient $(r)$ for each individual parameter, $k \sigma, k \ell$, and $m_{v}$, are also given. Of the three parameters, $k \ell$ had the largest RMSE. This is consistent with our previous discussion on the measurement uncertainty of the correlation length. Nevertheless, numeric values in Table 6 confirm the good performance of surface parameter inversion by model-trained DLNN with inputs from bistatic radar scattering measurements.

Table 6. Comparison of surface parameters between measured and inverted by model-trained DLNN.

\begin{tabular}{|c|c|c|c|c|c|c|c|c|}
\hline \multicolumn{3}{|c|}{$\begin{array}{c}\text { Truth } \\
\text { (Measured) }\end{array}$} & \multicolumn{3}{|c|}{$\begin{array}{c}\text { Inverted } \\
\text { (Network-Inverted) }\end{array}$} & \multicolumn{3}{|c|}{ Difference (Absolute) } \\
\hline$k \sigma$ & $k \ell$ & $m_{v}$ & $k \sigma$ & $k \ell$ & $m_{v}$ & $k \sigma$ & $k \ell$ & $m_{v}$ \\
\hline 1.05 & 2.51 & 0.16 & 1.24 & 2.48 & 0.17 & 0.19 & 0.03 & $0.59 \%$ \\
\hline 2.09 & 5.02 & 0.12 & 1.99 & 5.27 & 0.12 & 0.09 & 0.25 & $0.28 \%$ \\
\hline 2.62 & 6.28 & 0.11 & 2.83 & 8.74 & 0.11 & 0.21 & 2.46 & $0.41 \%$ \\
\hline 4.19 & 10.05 & 0.12 & 4.25 & 9.99 & 0.13 & 0.06 & 0.06 & $1.43 \%$ \\
\hline 5.23 & 12.56 & 0.10 & 7.49 & 12.56 & 0.09 & 2.25 & 0.004 & $0.11 \%$ \\
\hline 8.37 & 20.09 & 0.09 & 7.64 & 22.46 & 0.09 & 0.73 & 2.37 & $0.48 \%$ \\
\hline 0.20 & 1.00 & 0.87 & 0.19 & 1.10 & 0.94 & 0.006 & 0.10 & $6.57 \%$ \\
\hline \multirow[t]{2}{*}{3.28} & 29.60 & 0.07 & 3.10 & 32.98 & 0.06 & 0.18 & 3.38 & $0.34 \%$ \\
\hline & \multicolumn{2}{|c|}{$k \sigma$} & \multicolumn{4}{|c|}{$k \ell$} & \multicolumn{2}{|c|}{$m_{v}$} \\
\hline RMSE & \multicolumn{2}{|c|}{0.85} & RMSE & \multicolumn{2}{|c|}{1.70} & RMSE & \multicolumn{2}{|c|}{$2.41 \%$} \\
\hline nRMSE & \multicolumn{2}{|c|}{0.10} & nRMSE & \multicolumn{2}{|c|}{0.09} & nRMSE & \multicolumn{2}{|c|}{0.03} \\
\hline $\mathrm{r}$ & \multicolumn{2}{|c|}{0.95} & $\mathrm{r}$ & \multicolumn{2}{|c|}{0.99} & $\mathrm{r}$ & \multicolumn{2}{|c|}{0.99} \\
\hline
\end{tabular}

\section{Conclusions}

Radar-polarized bistatic scattering from rough soil surface was examined. Compared to backscattering, bistatic scattering provided richer scattering features in response to surface parameters, roughness, and moisture content. The angular patterns in hemispherical plots showed a strong coupling between radar scattering and surface parameters. Hence, the $4 \mathrm{Vs}$ (volume, variety, velocity, and veracity) in big data were persistently preserved in bistatic scattering behavior. However, the payoff is that, in terms of parameter retrieval, higher accuracy was attainable from bistatic observation. 
The task of rough-surface parameter (geometric and dielectric) retrieval from bistatic radar scattering data is a highly nonlinear problem. In this paper, it has been shown that the use of the Kalman filtering algorithm enhances the separability for highly nonlinear boundary problems. The Kalman filtering process is a recursive minimum mean square estimation procedure in which each updated estimate of the neural network weight is computed from the previous estimate and the new input data. Since there was no a priori information available to the data, the surface roughness and soil moisture inversion from fully bistatic radar scattering data by a Kalman filter-trained dynamic learning neural network were presented in this study. The network was able to tackle the complex inversion problem with very fast learning. Experimental testing showed good performance of surface parameter inversion by the model-trained DLNN with inputs from bistatic radar scattering measurements. In the future study, through the model simulation and the proposed inversion scheme, the optimal bistatic observation configuration (in the sense of most efficient multiple inputs-multiple outputs (MIMO) to come up with high retrieval accuracy) will be investigated.

Before closing, it should be emphasized that, to our best understanding, real bistatic radar measurements are scant, or only taken in very limited and yet specific radar configurations (e.g., specular direction). In this paper, we demonstrated how useful bistatic data is in retrieving multiple surface parameters of interest, as compared to the backscattering data which occupies almost all of the data available, at least currently. It is therefore our objective to promote the focus more on the bistatic scattering measurements, which are shown to be powerful in inferring the surface parameters.

Author Contributions: Y.Y. and K.-S.C. conceived and developed the scattering model and data model; Y.Y. performed and analyzed the numerical simulations; Y.Y. and K.-S.C. wrote and edited the paper; G.S. discussed and examined the numerical experiments and final results.

Funding: This work was supported by the grant 2017GJXGK024, and National Natural Science Foundation of China under the grant 41531175.

Conflicts of Interest: The authors declare no conflict of interest.

\section{References}

1. Tsang, L.; Kong, J.A.; Shin, R.T. Theory of Microwave Remote Sensing; Wiley-Interscience: New York, NY, USA, 1985.

2. Fung, A.K. Microwave Scattering and Emission Models and Their Applications; Artech House: Norwell, MA, USA, 1994.

3. Fung, A.K.; Chen, K.S. Microwave Scattering and Emission Models for Users; Artech House: Norwood, MA, USA, 2010.

4. Ulaby, F.T.; Long, D. Microwave Radar and Radiometric Remote Sensing; University of Michigan: Ann Arbor, MI, USA, 2014.

5. Schiavulli, D.; Nunziata, F.; Pugliano, G.; Migliaccio, M. Reconstruction of the normalized radar cross section field from GNSS-R delay-doppler map. IEEE J. Sel. Top. Appl. Earth Sci. Remote Sens. 2014, 7, 1573-1583. [CrossRef]

6. Zavorotny, V.U.; Voronovich, A.G. Scattering of GPS signal from the ocean with wind remote sensing application. IEEE Trans. Geosci. Remote Sens. 2000, 38, 951-964. [CrossRef]

7. Kim, S.B.; Moghaddam, M.; Tsang, L.; Burgin, M.; Xu, X.; Njoku, E.G. Models of L-band radar backscattering coefficients over global terrain for soil moisture retrieval. IEEE Trans. Geosci. Remote Sens. 2014, 52, 1381-1396. [CrossRef]

8. Kim, S.B.; van Zyl, J.J.; Johnson, J.T.; Moghaddam, M.; Tsang, L.; Colliander, A.; Dunbar, R.S. Surface soil moisture retrieval using the L-Band synthetic aperture radar onboard the soil moisture active-passive satellite and evaluation at core validation sites. IEEE Trans. Geosci. Remote Sens. 2017, 55, 1897-1914. [CrossRef]

9. Shao, W.Z.; Zhang, Z.; Li, X.M.; Wang, W.L. Sea surface wind speed retrieval from TerraSAR-X HH polarization data using an improved polarization ratio model. IEEE J. Sel. Top. Appl. Earth Sci. Remote Sens. 2016, 9, 4991-4997. [CrossRef] 
10. Neelam, M.; Colliander, A.; Mohanty, B.P.; Jackson, T.J.; Cosh, M.H.; Misra, S. Multi-scale surface roughness model for soil moisture retrieval. In Proceedings of the 2017 IEEE International Geoscience and Remote Sensing Symposium (IGARSS), Fort Worth, TX, USA, 23-28 July 2017; pp. 4963-4965.

11. Gelman, A.; Carlin, J.B.; Stern, H.S.; Dunson, D.B.; Vehtari, A.; Rubin, D.B. Bayesian Data Analysis, 3rd ed.; CRC Press: Boca Raton, FL, USA, 2013.

12. Tsang, L.; Chen, Z.X.; Oh, S.; Mark, R.J., II; Chang, A.T.C. Inversion of snow parameters from passive microwave remote sensing measurements by a neural network trained with a multiple scattering model. IEEE Trans. Geosci. Remote Sens. 1992, 30, 1015-1024. [CrossRef]

13. Dawson, M.S.; Olvera, J.; Fung, A.K.; Manry, M.T. Inversion of surface parameters using fast learning neural network. In Proceedings of the IGARSS'92, Houston, TX, USA, 26-29 May 1992; pp. 910-912.

14. Davis, D.T.; Chen, Z.X.; Tsang, L.; Hwang, J.N.; Chang, A.T.C. Retrieval of snow parameters by iterative inversion of a neural network. IEEE Trans. Geosci. Remote Sens. 1993, 31, 827-841. [CrossRef]

15. Chen, K.S.; Kao, W.L.; Tzeng, Y.C. Retrieval of surface parameters using dynamic learning neural network. Int. J. Remote Sens. 1995, 16, 801-809. [CrossRef]

16. Chen, K.S.; Wu, T.D.; Shi, J.C. A model-based inversion of rough soil surface parameters from radar measurements. J. Electromagn. Waves Appl. 2001, 15, 173-200. [CrossRef]

17. Thiria, S.; Mejia, C.; Badran, F. A neural network approach for modeling nonlinear transfer functions: Application for wind retrieval from space borne scatter meter data. J. Geophys. Res. Oceans 1993, 98, 22827-22841. [CrossRef]

18. Chen, K.S.; Tzeng, Y.C.; Chen, P.C. Retrieval of ocean winds from satellite scatter meter by a neural network. IEEE Trans. Geosci. Remote Sens. 1999, 37, 247-256. [CrossRef]

19. Hey, T.; Tansley, S.; Toll, K. The Fourth Paradigm: Data-Intensive Scientific Discovery, 1st ed.; Microsoft Research: Mountain View, CA, USA, 2009.

20. Chen, K.S.; Wu, T.D.; Tsang, L.; Li, Q.; Shi, J.C.; Fung, A.K. Emission of rough surfaces calculated by the integral equation method with comparison to three-dimensional moment method simulations. IEEE Trans. Geosci. Remote Sens. 2003, 41, 90-101. [CrossRef]

21. Yang, Y.; Chen, K.S.; Tsang, L.; Yu, L. Depolarized backscattering of rough surface by AIEM model. IEEE J. Sel. Top. Appl. Earth Sci. Remote Sens. 2017, 10, 4740-4752. [CrossRef]

22. Fung, A.K.; Li, Q.; Chen, K.S. Backscattering from a randomly rough dielectric surface. IEEE Trans. Geosci. Remote Sens. 1992, 30, 356-369. [CrossRef]

23. Chen, K.S.; Wu, T.D.; Fung, A.K. A Note on the multiple scattering in an IEM model. IEEE Trans. Geosci. Remote Sens. 2000, 38, 249-256. [CrossRef]

24. Huang, S.; Tsang, L. Electromagnetic scattering of randomly rough soil surfaces based on numerical solutions of Maxwell equations in three-dimensional simulations using a hybrid UV/PBTG/SMCG method. IEEE Trans. Geosci. Remote Sens. 2012, 50, 4025-4035. [CrossRef]

25. Huang, S.; Tsang, L.; Njoku, E.G.; Chen, K.S. Backscattering coefficients, coherent reflectivities, and emissivities of randomly rough soil surfaces at L-band for SMAP applications based on numerical solutions of Maxwell equations in three-dimensional simulations. IEEE Trans. Geosci. Remote Sens. 2010, 48, 2557-2568. [CrossRef]

26. Liao, T.H.; Tsang, L.; Huang, S.; Niamsuwan, N.; Jaruwatanadilok, S.; Kim, S.B.; Ren, H.; Chen, K.L. Co-polarized and cross-polarized backscattering from random rough soil surfaces from L-band to Ku-band using numerical solutions of Maxwell's equations with near-field precondition. IEEE Trans. Geosci. Remote Sens. 2016, 54, 651-662. [CrossRef]

27. Tsang, L.; Ding, K.H.; Huang, S.W.; Xu, X.L. Electromagnetic computation in scattering of electromagnetic waves by random rough surface and dense media in microwave remote sensing of land surfaces. Proc. IEEE 2013, 101, 255-279. [CrossRef]

28. Oh, Y.; Sarabandi, K.; Ulaby, F.T. An empirical model and an inversion technique for radar scattering from bare soil surfaces. IEEE Trans. Geosci. Remote Sens. 1992, 30, 370-381. [CrossRef]

29. Hallikainen, M.T.; Ulaby, F.T.; Dobson, M.C.; El-Rayes, M.A.; Wu, L.K. Microwave dielectric behavior of wet soil-Part I: Empirical models and experimental observation. IEEE Trans. Geosci. Remote Sens. 1985, GE-23, 25-34. [CrossRef]

30. Dobson, M.C.; Ulaby, F.T.; Hallikainen, M.T.; El-Rayes, M.A. Microwave dielectric behavior of wet soil-Part II: Dielectric mixing models. IEEE Trans. Geosci. Remote Sens. 1985, GE-23, 35-46. [CrossRef] 
31. Zeng, J.Y.; Chen, K.S.; Bi, H.Y.; Zhao, T.J.; Yang, X.F. A comprehensive analysis of rough soil surface scattering and emission predicted by AIEM with comparison to numerical simulations and experimental measurements. IEEE Trans. Geosci. Remote Sens. 2017, 55, 1-13. [CrossRef]

32. Çetin, M.; Karl, W.C. Feature-Enhanced Synthetic Aperture Radar Image Formation Based on Nonquadratic Regularization. IEEE Trans. Image Process. 2001, 10, 623-631. [CrossRef]

33. Tzeng, Y.C.; Chen, K.S.; Kao, W.L.; Fung, A.K. A dynamic learning neural network for remote sensing applications. IEEE Trans. Geosci. Remote Sens. 1994, 32, 1096-1102. [CrossRef]

34. Scharf, L.L. Statistical Signal Processing-Detection, Estimation, and Time-Series Analysis; Addison Wesley Publishing Co.: Reading, MA, USA, 1991.

35. Hauck, B.; Ulaby, F.; DeRoo, R. Polarimetric bistatic measurement facility for point and distributed targets. IEEE Antennas. Propag. Mag. 1998, 40, 31-41. [CrossRef]

36. Nashashibi, A.Y.; Ulaby, F.T. MMW polarimetric radar bistatic scattering from a random surface. IEEE Trans. Geosci. Remote Sens. 2007, 45, 1743-1755. [CrossRef]

37. Macelloni, G.; Nesti, G.; Pampaloni, P.; Sigismondi, S.; Tarchi, D.; Lolli, S. Experimental validation of surface scattering and emission models. IEEE Trans. Geosci. Remote Sens. 2000, 38, 459-469. [CrossRef]

38. Brogioi, M.; Pettinato, S.; Macelloni, G.; Paloscia, S.; Pampaloni, P.; Pierdicca, N.; Ticconi, F. Sensitivity of bistatic scattering to soil moisture and surface roughness of bare soils. Int. J. Remote Sens. 2010, 31, 4227-4255. [CrossRef]

(C) 2019 by the authors. Licensee MDPI, Basel, Switzerland. This article is an open access article distributed under the terms and conditions of the Creative Commons Attribution (CC BY) license (http://creativecommons.org/licenses/by/4.0/). 